\title{
Vibrational tests of a system with two coupled beams and a distributed tip mass
}

\author{
Andrzej Rysak ${ }^{1, *}$, Euan Rodgers ${ }^{2}$, and Grzegorz Litak ${ }^{1,3}$ \\ ${ }^{1}$ Lublin University of Technology, Faculty of Mechanical Engineering, Nadbystrzycka 36, 20-618 \\ Lublin, Poland \\ ${ }^{2}$ Heriot-Watt University, School of Engineering \& Physical Sciences, Edinburgh, Scotland, UK EH14 \\ $4 \mathrm{AS}$ \\ ${ }^{3}$ AGH University of Science and Technology, Faculty of Mechanical Engineering and Robotics, De- \\ partment of Process Control, Mickiewicza 30, 30-059 Krakow, Poland
}

\begin{abstract}
We present the dynamical response of a nonlinear system designed for vibrational energy harvesting. The mechanical resonator is composed of two steel beams. The beams are coupled by a screw together at the ends with various distance element. Additionally, two piezoelectric patches are placed on their surface giving output powers on electric loads. In the article, we examine the output powers and dynamics of the system for variable frequency, different sizes of distance elements and several tip mass distributions.
\end{abstract}

\section{Introduction}

Recently, various nonlinear systems where proposed for vibration energy harvesting [1-4]. They guarantee broadband frequency energy converters important for variable vibration energy sources [5-7] comparing to linear devices [8]. The role of nonlinearities [5, 6] is important non only in broadening of frequency in the main resonance region through inclination of the resonance curve but also in involving multiple solutions. Larger amplitude solutions were observed in the double well system resonators $[6,9]$. Following the concept of the inverted elastic pendulum which undergoes a buckling bifurcation of single to double equilibria under load of a tip mass $[10,11]$, we propose the system of specific elasticity based on the coupled beams and a tip mass. In this system we observe buckling phenomenon for larger amplitudes of excitation.

The tested system is characterized by a simple construction. The two flexible beams are coupled with a spacer. In addition, the end of the system is loaded with a tip mass. The separation distance and the value and distribution of tip mass can be changed in particular measurements. To our knowledge, the beam arrangement with this geometry has not been studied before. The construction of the system creates conditions to excite high amplitude vibrations at various modes involving snap through and impact phenomena. Therefore, the main aim of the work is to examine the dynamics of this system in various configurations and at different levels of excitation. The dynamics of both beams is studied by measuring the average relative power generated in the piezoelectric elements.

\footnotetext{
*e-mail: a.rysak@pollub.pl
} 


\section{Experiment configuration}

The geometry of the investigated system is presented in Fig. 1 1. Two beams are fixed to the shaker base with a constant distance $D$.

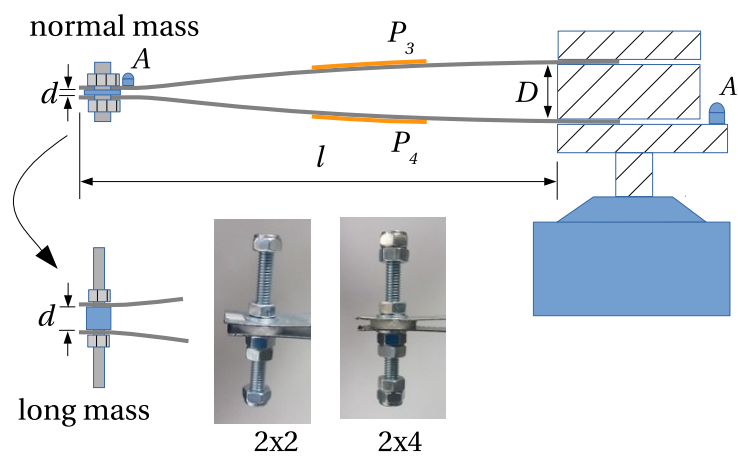

Figure 1. System geometry. $P_{3}, P_{4}$ - top and bottom patches (we will use the same notation for power output on the corresponding electrical resistors $R_{3}$ and $R_{4}$ ). $d$ - spacer thickness. Difference between normal and long masses is shown. The photos show the configurations of the long tip mass with two and four additional nuts screwed to its ends, marked as $2 \times 2$ and $2 \times 4$, respectively

Both beams were coupled through a distance element with thickness $d$ (Fig. 1). A piezoelectric patch was sticked to each beam. Their ends are screwed with a spacer of thickness $d$ between them. The screw joining the ends of both beams is simultaneously the tip mass of the system. In the measurements, we use two tip masses: normal (short) and long, both with the same value $m=17.95 \mathrm{~g}$. In addition, at the ends of the long mass we screw the pairs of nuts, obtaining new configurations of tip mass: $2 \times 2$ and $2 x 4$, as it was shown in figure 1 . Such determinations of the tip mass are related to the weight of a single nut, which equals to about $2 g$

The scheme of the measurement system is shown in Fig. 3. A series of measurements of the system were made at various distances $d$, tip masses and levels of excitation. There was no stimulation control in the measurements. Therefore, to minimize the effect of variable excitation on the measurement results, all measured values have been normalized. For this purpose, the following conversions were made. Firstly, the window was moved along the time axis with discrete values corresponding to the measuring points. The width of the window equals $n$ points on time axis and the length of the shift step is $\Delta t_{s}$. Next, for each window was calculated the square of the standard deviation of the excitation $\sigma_{a}^{2}$ which was expressed in $\left(\mathrm{m} / \mathrm{s}^{2}\right)^{2}$. For both piezo voltages, the average normalized electrical power in the window was calculated according to the formula:

$$
\bar{P}=\frac{1}{n R \sigma_{a}^{2}} \sum_{i=1}^{n} U_{i}^{2},
$$

where $\sigma_{a}$ is a standard deviation of excitation acceleration calculated for $n$ steps.

\section{Results}

In order to gain an insight into the dynamics of the tested system, we present comparative results that illustrate the impact of changing the various parameters of the experiment on 


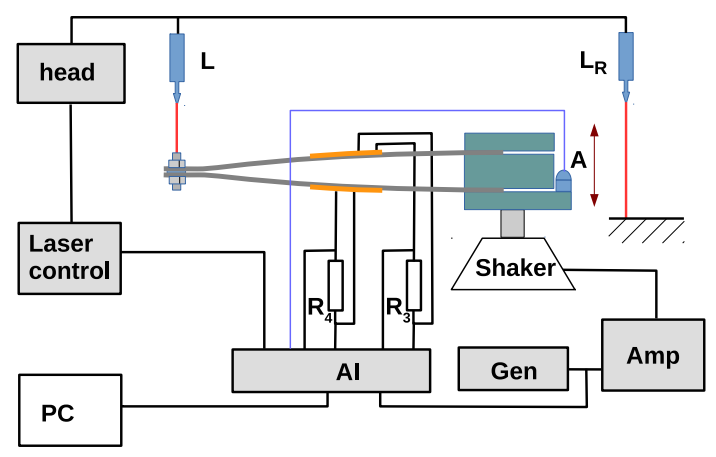

Figure 2. Scheme of the experiment. $L$ and $L_{R}$ the reference laser system to velocity measurements. Gen - waveform generator, $A m p$ - shaker amplifier, $A I$ - input of data acquisition card, $A$ - accelerometer. The $R_{3}$ and $R_{4}$ resistors build voltage dividers which fit measured voltages to the data acquisition card input range

its vibrations end efficiency. Figure 3 shows the change in normalized powers under the influence of the change of the excitation level (a-b), the tip mass distribution (a-c), and the distance $d$.
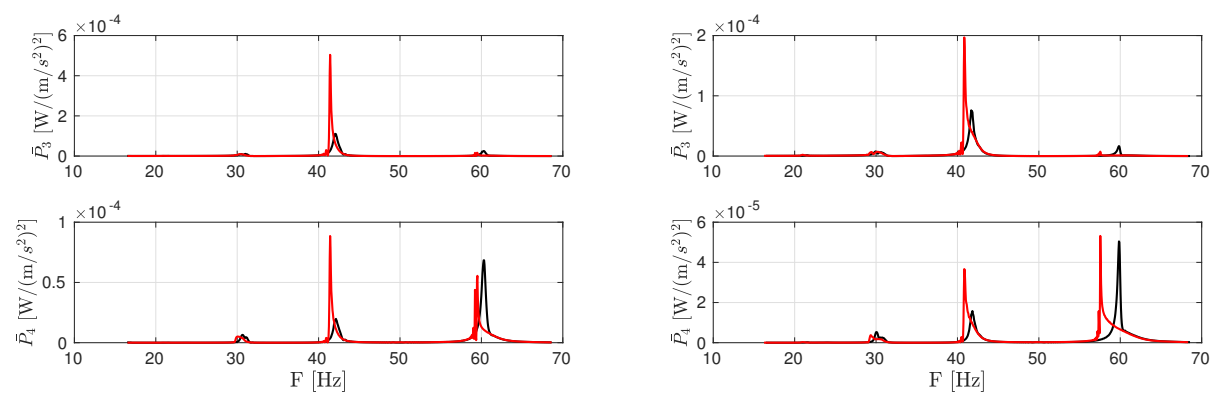

(a)

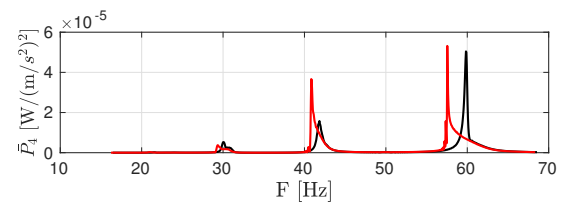

(b)

Figure 3. Comparing of results for two-way sweeps for power output (denoted by $P_{3}$ and $P_{4}$ corresponding to the top and bottom piezoelectrics - Fig. 1) obtained with referencing to the base case (a) with $d=1.9 \mathrm{~mm}$, normal mass, and $U_{E}=50 \mathrm{mV}$. In the right-side figure (b) both curves were measured for the excitation increased to the level $U_{E}=100 \mathrm{mV}$ of voltage generator. Figures in the lower row present results collected at $U_{E}=50 \mathrm{mV}\left(U_{E}\right.$ is voltage on a harmonic signal generator controlling the amplitude of excited vibrations). Figure (c) shows results obtained for the long mass, $d=1.9 \mathrm{~mm}$, and (d) for the long mass and $d=3.8 \mathrm{~mm}$. Black color - sweep up (towards higher frequencies), red color sweep down

Based on the results (a-b), it can be concluded that increasing the level of excitation reduces the relative efficiency of the system. With increased excitation, nonlinear effects are amplified, which is clearly visible in (b) as the $P_{4}$ power hysteresis in the resonance of around $60 \mathrm{~Hz}$. The change of the final weight distribution from normal to long (a-c) clearly modifies the vibration modes of both beams. This is visible in both resonant ranges: $44 \mathrm{~Hz}$, and $60 \mathrm{~Hz}$. But if for the long tip mass the distance $d$ increases, on can notice the equalization of the dynamics of both beams and shift of resonance areas towards the lower frequencies. The clearly visible discrepancies between up and down seep maxima are interesting. They 

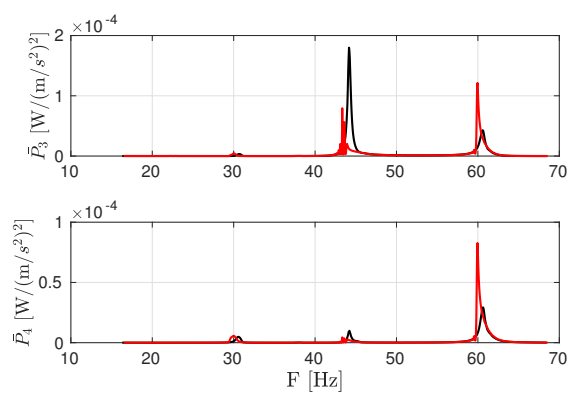

(c)

Figure 3. Continuation
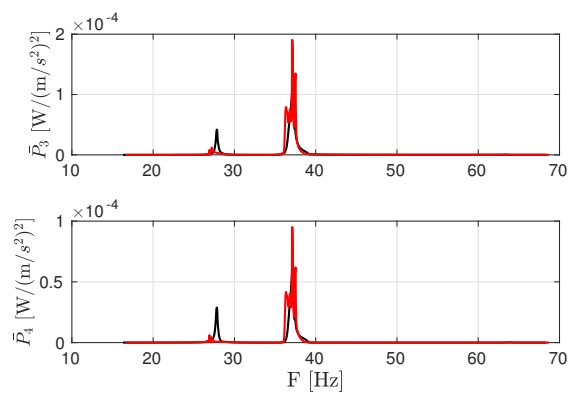

(d)

indicate the nonlinear effects. The assumed measuring procedure of solution continuation leads to dynamical hysteresis.

To explore these differences we repeated experiments for various system parameters. The next figure (Fig. 4) shows how the power generated in the system is modify under the influence of a small change in the tip mass distribution.
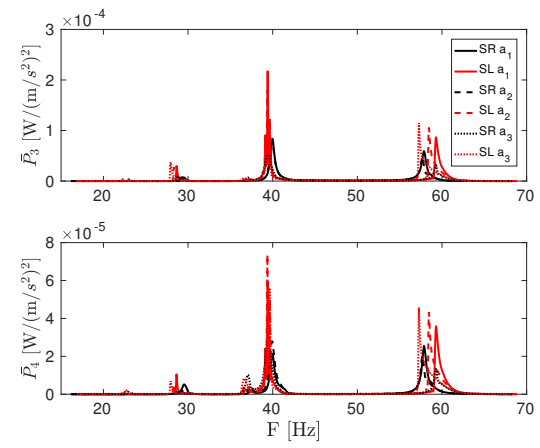

(a)
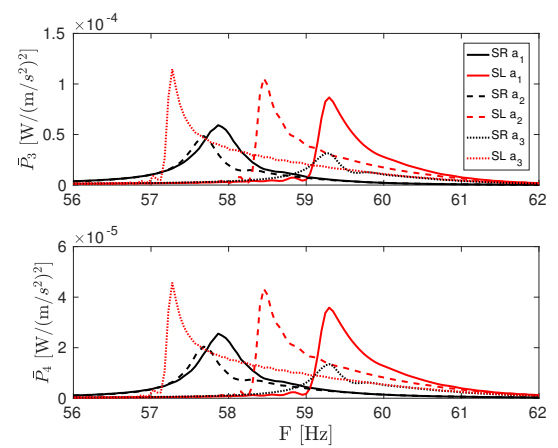

(b)

Figure 4. The influence of tip mass distribution on system power. In both cases: $d=3.0 \mathrm{~mm}$. Lines distinguish between different levels of excitation: line - $U_{E}=50 \mathrm{mV}$, dashed line $-U_{E}=75 \mathrm{mV}$, and doted line - $U_{E}=100 \mathrm{mV}$. (a,b) - tip mas $2 \times 2$, (c,d) - tip mas $2 \times 4$. Black color - sweep up, red color sweep down

In these measurements, we examine the system with $d=3.0 \mathrm{~mm}$ excited at the level of $U_{E}=50 \mathrm{mV}$. Both presented results differ in tip mass configuration: (a) $-2 x 2$, (c) $-2 x 4$ (see definition in Fig. 1). Figures (b) and (d), for both cases, show the magnification of the resonance area near $60 \mathrm{~Hz}$. A slight modification of the tip mass induces clear changes in the system vibrations. A new resonance occurs for a frequency of around $30 \mathrm{~Hz}$. At higher frequencies, two effects can be noted for the resonance area of $60 \mathrm{~Hz}$. Firstly, in both piezoelectric elements there is a large increase in normalized power, mainly for the sweeping towards lower frequencies (red). The second effect is the shift of resonance peaks towards the lower frequencies, which is definitely greater for the red lines - the direction of the sweep to the left (red). To make it easier to observe this phenomenon, the resonance area around $60 \mathrm{~Hz}$ was shown in zoom in figures $4 \mathrm{~b}$ and $\mathrm{d}$. 

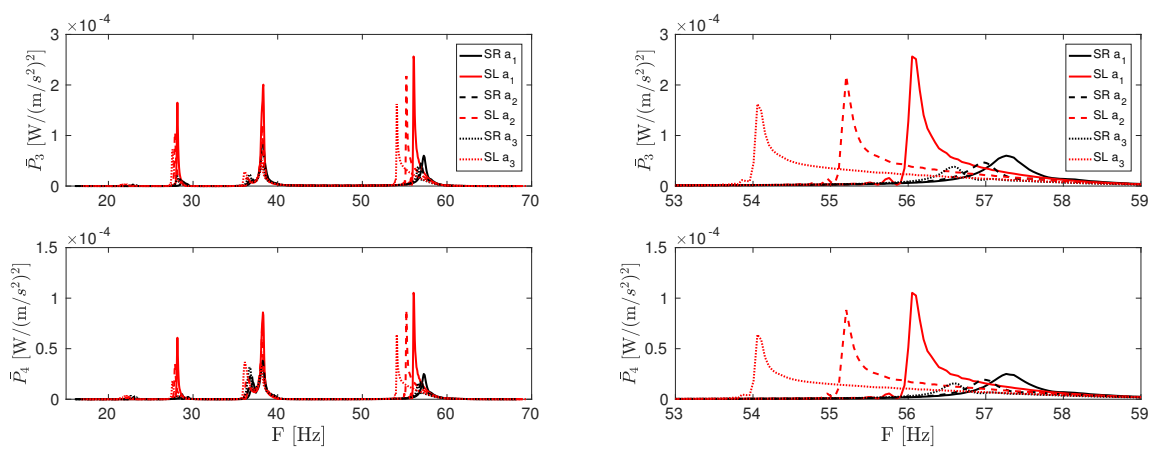

(c)

(d)

Figure 4. Continuation

Distance $d$ is an important parameter, significantly affecting the dynamics of the system. Interestingly, due to the geometrical conditions (see Fig. 1), the distance $d$ play some role in the contact appearance and loss during buckling of one of the beam. These impacting solution can give fairly large power for higher frequency [12].

The results of the system dynamics study for different values of this parameter are presented in Fig. 5. It shows the results obtained for highest excitation on the level of $U_{E}=100 \mathrm{mV}$. Figure 5 contains two three-dimensional plots showing the power charac-

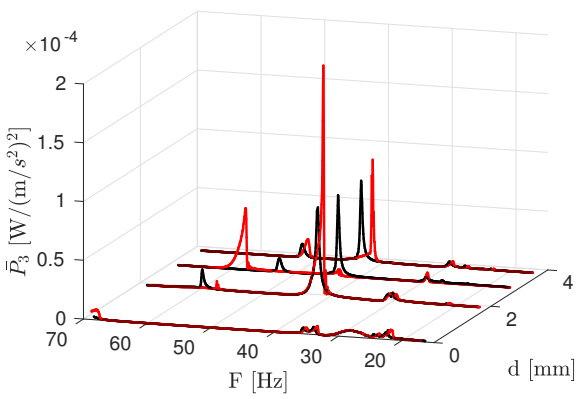

(a)

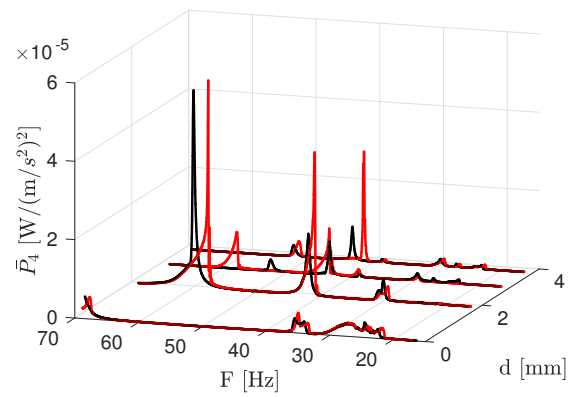

(b)

Figure 5. The influence of distance $d$ on system power generation and hysteresis. In both cases $U_{E}=$ $100 \mathrm{mv}$, and normal mass was used. (a) $P_{3}$ power, (b) $P_{4}$ power (See Fig. for definition of $P_{3}$ and $P_{4}$ ). Black color - sweep up, red color - sweep down

teristics of both piezoelectric elements obtained for different $d$ values. Both plots show the variability of peak voltage values, frequency in which they occur and hysteresis of resonances which appears when changing the sweep direction. Both 3D plots show a clear gain in normalized power for $d=1.9 \mathrm{~mm}$. Because in our experiment the values of $d$ are rarely distributed, one can probably find the optimal distance $d$, for which the system's efficiency will be the highest. 


\section{Discussion and conclusions}

The presented results were selected from the many obtained from the measurements of the studied system for different values of the experiment parameters. They focus mainly on the assessment of changes in the dynamics and efficiency of the system caused by changes in the tip mass distribution and distance $d$. Based on the obtained results, we can conclude that by modifying these parameters, one can tune the system to the expected modes of operation. Along with such modifications, a strong nonlinear effects appear which cause response difficult to predict. Nonlinear effects frequently improve the system's performance by broadening of the resonance area and by including new solutions for a higher excitation level. Another possibility is minor changes to one of the main parameters or the state variable via disturbances can improve energy harvesting considerably.

This work was supported by the Polish National Science Center under grant Agreement No. DEC2013/11/D/ST8/03308.

\section{References}

[1] Harne, R.L., Wang, K.W., Smart Mat. Struct. 22, 023001 (2013)

[2] Pellegrini, S.P., Tolou, N., Schenk, M., Herder, J.L., J. Intell. Mater. Syst. Struct. 24, 1303-1312 (2013)

[3] Twiefel, J., Westermann, H., J. Intell. Mater. Syst. Struct. 24, 1291-1302 (2013)

[4] Litak, G., Manoach, E., Halvorsen, E., Eur. Phys. J.-Special Topics 224, 2671-2673 (2015)

[5] Daqaq, M.F., Masana, R., Erturk, A., Quinn, D.D., Applied Mechanics Reviews 66, 040801 (2014).

[6] Huguet, T., Badel, A., Lallart, M., Appl. Phys. Lett. 111, 173905 (2017)

[7] Wolszczak, P., Lygas, K., Litak, G., Mech. Sys. Sign. Process. 107, 43-52 (2018)

[8] Beeby, S.P., Tudor, M.J., White, N.M., Meas. Sci. Technol. 17, R175-R195 (2006)

[9] Syta, A., Litak, G., Friswell, M.I., Adhikari, S., Eur. Phys. J. B 89, 99 (2016).

[10] Friswell, M.I., Ali, S.F., Adhikari, S., Lees, A.W., Bilgen, O., Litak, G., J. Intell. Mater. Syst. Struct. 23, 1505-1521 (2012).

[11] Erturk, A., Hoffmann, J., Inman, D.J., Appl. Phys. Lett. 94, 254102 (2009)

[12] Stoykov, S., Manoach, E., Litak, G., Eur. Phys. J.-Special Topics 224, 2755-2770 (2015). 